\title{
Utilizing Upper Arm Images in Determining Body Mass Index and Nutritional Status
}

\author{
Sami F. Al-Mashaqbah \\ Dept. of Biomedical Systems and \\ Medical Informatics, Hijjawi Faculty for \\ Engineering Technology, Yarmouk \\ University, Irbid, Jordan \\ sami.m@yu.edu.jo
}

\author{
Walid Al-Zyoud \\ Biomedical Engineering Dept., \\ School of Applied Medical Sciences, \\ German Jordanian University, \\ Amman, Jordan \\ walid.alzyoud@gju.edu.jo
}

\author{
Awad Al-Zaben \\ Dept. of Biomedical Systems and \\ Medical Informatics, Hijjawi Faculty for \\ Engineering Technology, Yarmouk \\ University, Irbid, Jordan \\ azaben@yu.edu.jo
}

\begin{abstract}
Body mass index (BMI) is the most widely used index for determining the fitness status of an individual. Midd-upper arm circumference (MUAC) is another commonly used anthropometric index, providing a simple measurement widely used for nutrition level screening. However, building a massive population database based on both BMI and MUAC measurements is time-consuming and may introduce errors due to instrumentations and different operators. In this paper, a measurement technique of BMI from photographic images of the upper arm is presented. Three parameters are measured from the subject photos with a scale attached, and these are upper arm length, sagittal plane middle arm thickness (SMT), and frontal plane middle thickness (FMT). Different parameters are derived from the SMT and the FMT. These parameters are used to study the correlation and measurements agreements with the BMI. It is found that all parameters related to SMT correlate very well with the BMI with correlation coefficients of more than $90 \%$. In addition, the SMT measurement limits (both upper and lower) are acceptable and within the clinical significance range. Finally, the receiver operating characteristics analysis of both SMT and MUAC has been evaluated. The findings show that SMT is slightly better than MUAC since it gives excellent sensitivity $(94.7 \%)$ without compromising the specificity $(88.5 \%)$ when compared to the MUAC results.
\end{abstract}

\section{Keywords-BMI; obesity; sagittal plane}

\section{INTRODUCTION}

As technology increased our quality of life, it led to a sedentary lifestyle and increased the prevalence of overweight or obesity in a global scale [1]. There is a metric difference between overweight and obesity in terms of the mass of the human body. According to the World Health Organization (WHO), obesity is defined as a medical condition where the fat mass of the human body is excess to the point that might negatively affect health. Overweight is being fat in a degree between healthy and obese. The association between obesity and the increased risk of developing a wide range of chronic diseases such as cardiovascular diseases, diabetes mellitus, and even cancer has been reported and raised public health concerns [2-4]. Currently, one of the most common methods to classify people based on their anthropometric (height and weight) characteristics is the body mass index (BMI), an index that reflects an individual's body fatness [5]. BMI is calculated by dividing the body mass in kilogram by the squared body height in meters [6]. The WHO obesity classification is based on BMI [7] which is widely adopted [8]. Four categories have been adopted: underweight: if the measured BMI is in the range 15-19.9, normal: if it is in the range 20-24.9, overweight: if it is in the range 25-29.9, and obese if it is more than 30 . There are other obesity classification systems with alternative terms. For example, the one suggested in [9], was not similar to the WHO-BMI system in terms of the categories cutoff points. In 1997, the obese category has been divided by the International Obesity Task Force into three categories as shown in Table I [10].

TABLE I. BMI CATEGORIES [10]

\begin{tabular}{|c|c|}
\hline Categories & BMI Range \\
\hline Underweight & $15-19.9$ \\
\hline Normal weight & $20-24.9$ \\
\hline Overweight & $25-29.9$ \\
\hline Obesity & \\
\hline Class I & $30-34.9$ \\
\hline Class II & $35-39.9$ \\
\hline Class III & $\geq 40$ \\
\hline
\end{tabular}

Obesity is the term describing excessive accumulated amount of triacylglycerols in the adipose tissue of the human body. From an anatomical and physiological point of view, obesity is called adiposity. Adiposity studies on public health are dominantly based on population surveys, something that makes BMI as a direct fat indicator quite debatable due to differences in variables including gender, age, ethnic group, and race [11-13]. It is worth mentioning that fat accumulation in different areas of the human body might be related to several pathophysiological indicators including inflammations, metabolic disorders, stress, and cardiovascular dysfunctions [14]. Despite this limitation, BMI is still the most widely used index for determining one's fitness status. On the other hand, the MUAC, which is another commonly used anthropometric index, provides a simple measurement that is widely used for nutrition level screening. MUAC has been used as a nutritional index in elderly, impatient, infants, children, university students, and pregnant women [15-19]. However, building a massive database of different populations to be used in 
determining the nutrition status of the public based on both BMI and MUAC measurements is time-consuming. Furthermore, these techniques may introduce errors due to instrumentations and different operators. In this study, a novel imaging approach is proposed in order to study the association between the BMI and anthropometric measurements of the upper arm, to find the best parameters that can be used to represent BMI in the sense of correlation and measurement agreement, and to compare the sensitivity and specificity of these parameters to MUAC. This method can be used in cases of disabilities where it is difficult to perform traditional measurements of height and weight. Furthermore, it provides a fast way to find BMI at temporary refugee camps and whenever the needed instruments are not available.

\section{DATABASE AND METHOD}

Photos of the subject in three positions are taken: sagittal plane position with the elbow flexed at $90^{\circ}$ with their palm facing upward, sagittal plane with their hands at the sides, and frontal plane with their hands at the sides as shown in Figure 1.
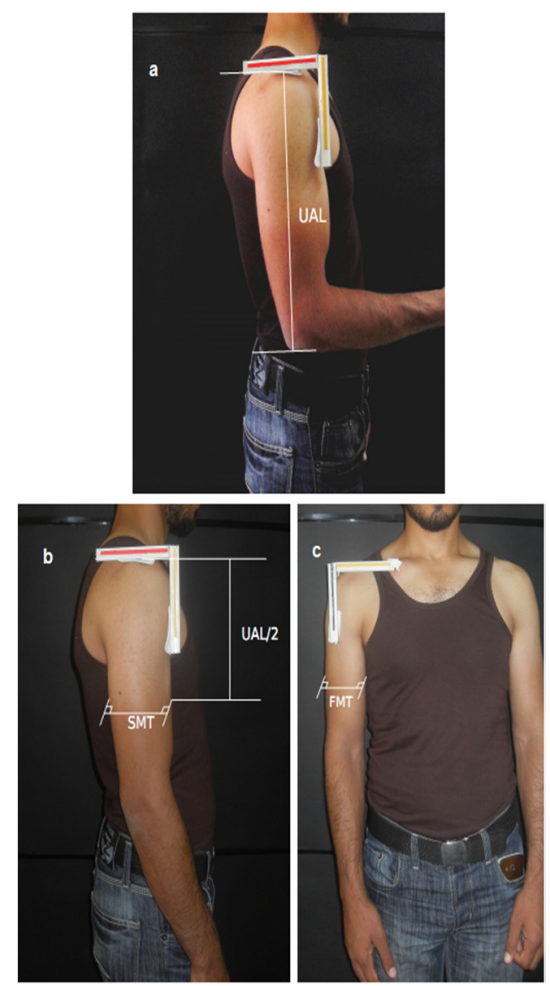

Fig. 1. (a) Standing on the sagittal plane with the elbow flexed at $90^{\circ}$ with palm facing upward, (b) standing on the sagittal plane with hands at the sides, (c) frontal plane with hands at the sides.

The upper arm length is measured relatively to the scale used in the photographic setup. Two parameters are then measured from the two other relative photos. These are the sagittal plane middle thickness (SMT) and the frontal plane middle thickness (FMT). The measured parameters are used to derive a set of parameters shown in Table II. In addition, subjects were asked to wear sleeveless shirts on top and to be barefoot. A digital weight scale was used to measure the weight of each subject. The height of the subjects was measured using a measuring tape attached to the wall while the subject stands on the floor at a right angle to the wall. While their right elbow was flexed at $90^{\circ}$, a measuring tape was used to measure the distance between the lateral tip of the acromion and the most distal point on the olecranon, which is considered as the upper arm length (UAL). The mid-upper arm was located and marked using a pen. A flexible measuring tape was used to measure the mid-arm circumference (MUAC) at the marked mid-point. A vernier was used to measure the SMT and the FMT. After taking informed consent, 45 healthy young male individuals with an average age of 22.25 years, ranging from 19.5 to 26.58 , participated in this study. Among them, 26 subjects were normal weighted with a BMI between 18 and 25 , ten subjects were over weighted with BMI between 25 and 30 , and nine subjects were obese with a BMI greater than 30 .

TABLE II. CALCULATED PARAMETERS

\begin{tabular}{|c|c|c|}
\hline Parameter & Description & Equation \\
\hline Pcirc & $\begin{array}{l}\text { Mid-arm circular perimeter } \\
\text { based on sagittal middle and } \\
\text { frontal middle thicknesses. }\end{array}$ & $\pi \frac{(\mathrm{SMT}+\mathrm{FMT})}{2}$ \\
\hline \multirow{2}{*}{ Pellip } & \multirow{2}{*}{$\begin{array}{l}\text { Mid-arm elliptical perimeter } \\
\text { based on sagittal middle and } \\
\text { frontal middle thicknesses. }\end{array}$} & \multirow{2}{*}{$2 \pi$} \\
\hline & & \\
\hline AcircSagittal & $\begin{array}{c}\text { Mid-arm circular cross- } \\
\text { sectional area based on sagittal } \\
\text { middle thickness. }\end{array}$ & $\pi\left(\frac{S M T}{2}\right)^{2}$ \\
\hline \multirow{2}{*}{ Acirc } & \multirow{2}{*}{$\begin{array}{l}\text { Mid-arm circular cross- } \\
\text { sectional area based on sagittal } \\
\text { middle and frontal middle } \\
\text { thicknesses. }\end{array}$} & \multirow{2}{*}{$\pi\left(\frac{S M T+F M T}{4}\right)^{2}$} \\
\hline & & \\
\hline Aellip & $\begin{array}{l}\text { Mid-arm elliptical cross- } \\
\text { sectional area based on sagittal } \\
\text { middle and frontal middle } \\
\text { thicknesses. }\end{array}$ & $4 \pi\left(\frac{\mathrm{SMT}}{2}\right)\left(\frac{\mathrm{FMT}}{2}\right)$ \\
\hline
\end{tabular}

\section{RESULTS}

The actual BMI is calculated from the well-known relation of the measured weight and height. This BMI is compared to the parameters measured and derived (Table II) after scaling. The null hypothesis is that the two measurements are not linearly related. Figure 2 shows the correlation analysis and the Bland-Altman plot $[20,21]$ of the measured MUAC versus BMI. The correlation analysis and the Bland-Altman plots of the sagittal plane parameters versus BMI are shown in Figure 3. Similarly, Figure 4 shows the correlation analysis and the Bland-Altman plots of the other parameters derived from both sagittal and frontal images versus the BMI.

Figure 5 shows the receiver operating characteristics of MUAC and SMT. The corresponding optimal cutoff-point analysis is shown in Figure 6. The results show an area under the curve (AUC) of 0.973 and 0.966 for MUAC and SMT, respectively. Furthermore, the optimal cutoff point of overweight for SMT is $11.2 \mathrm{~cm}$ with a sensitivity of $94.7 \%$ and specificity of $88.5 \%$, while the optimal cutoff point of overweight for MUAC is $30 \mathrm{~cm}$ with a sensitivity of $100 \%$ and specificity of $84.7 \%$. 
(a)

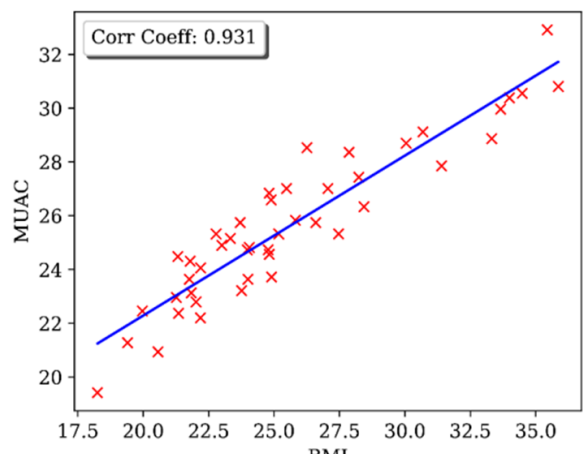

(b)

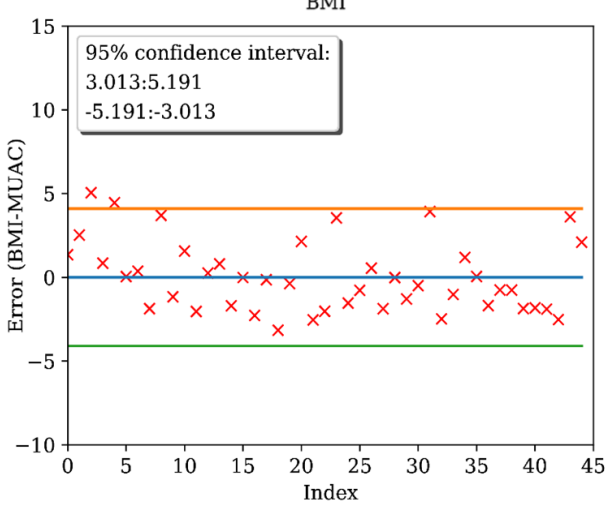

\section{DISCUSSION}

The current work aimed to investigate the usefulness of using images of the upper arm in determining BMI and nutritional status. Several parameters have been measured and derived from the images of the sagittal and frontal planes of the upper arm. These parameters' relationship to BMI is evaluated using two statistical measures: the correlation coefficient and the measurement agreement presented by Bland-Altman plots. The correlation coefficient values revealed a solid relationship between BMI and all parameters. The correlation coefficients were $0.926,0.936,0.855,0.916,0.916$, and 0.906 for SMT, AcircSagittal, Pcirc, Acirc, Pellip, and Aellip respectively. The best correlation corresponds to these parameters derived from a sagittal image (SMT and AcircSagittal). However, the correlation is between one variable and another, not the differences. Hence it is not recommended as a standalone method for assessing the comparability between methods [20]. Therefore, Bland-Altman plots were used to describe the agreement between each parameter and BMI. In the BlandAltman plot, the difference between the paired measurements is plotted against the mean of the two measurements [20,21]. The result shows that the SMT measurement agreement with BMI is better than with other parameters.

Fig. 2. Correlation analysis and Bland-Altman plot of MUAC versus BMI.

(a)

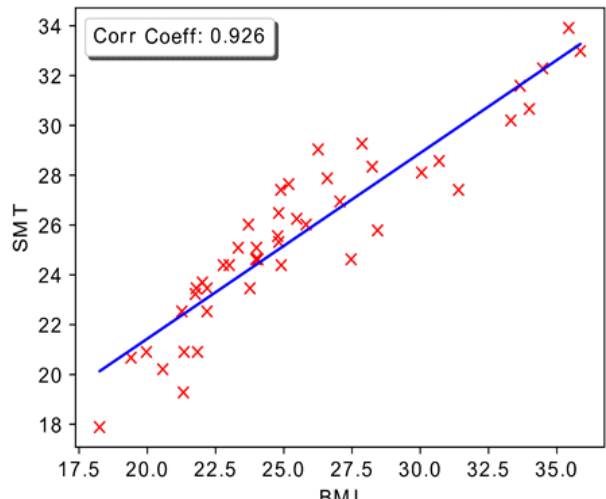

(b)

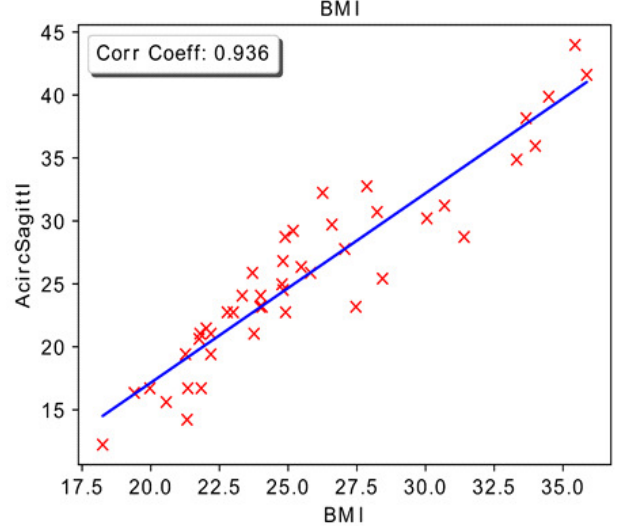

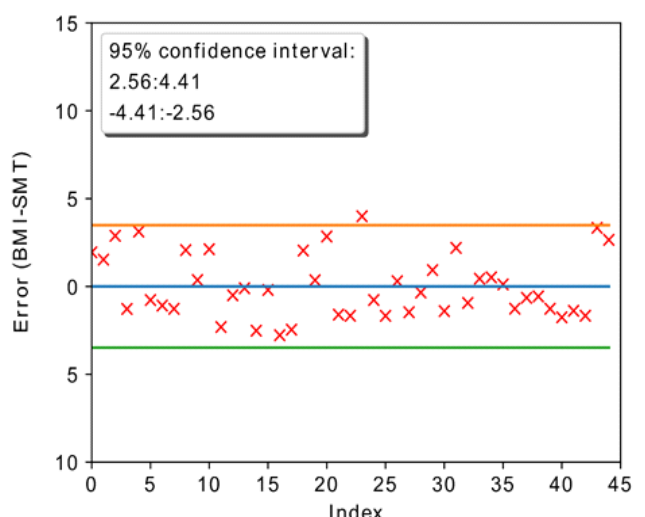

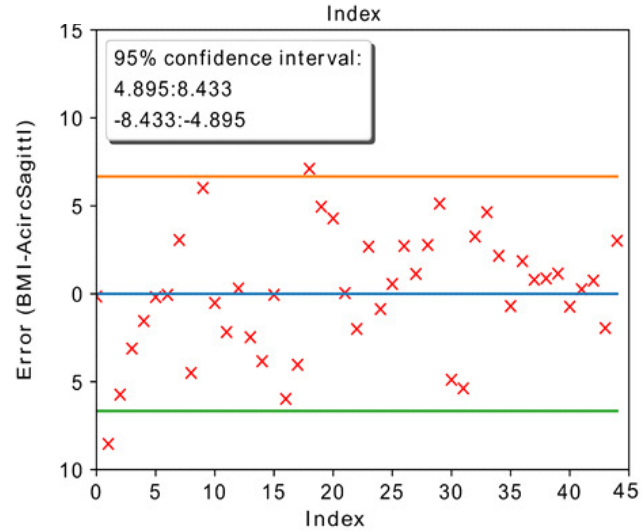

Fig. 3. Correlation analysis and Bland-Altman plots of parameters derived from sagittal plane: (a) SMT, (b) AcircSagittl 
(a)

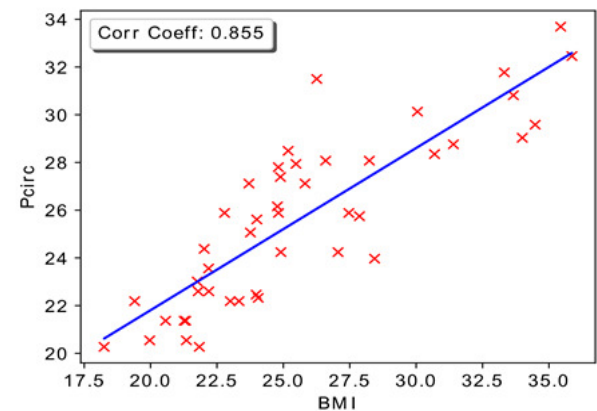

(b)

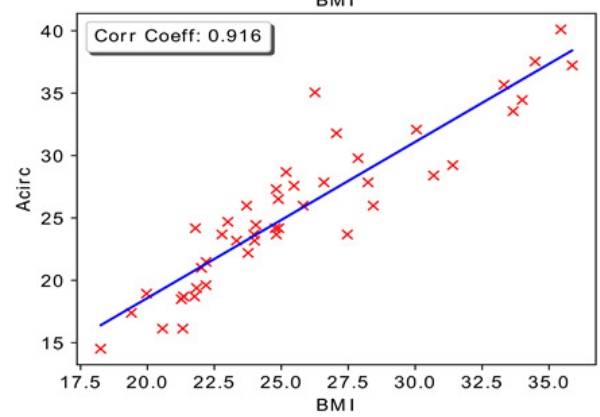

(c)

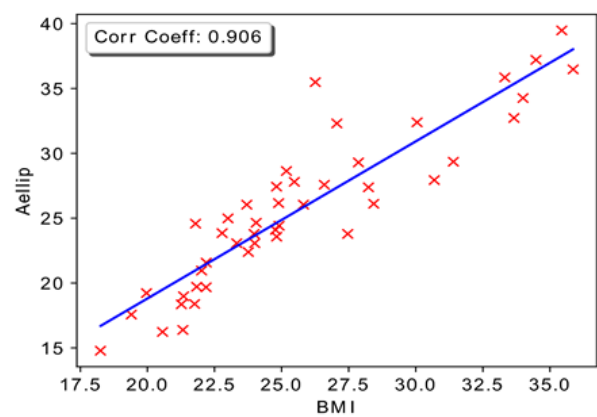

(d)

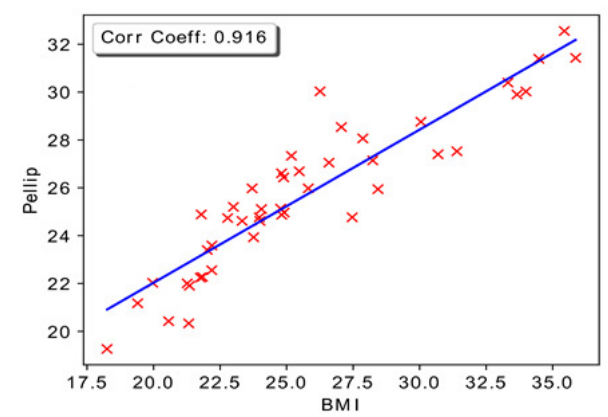

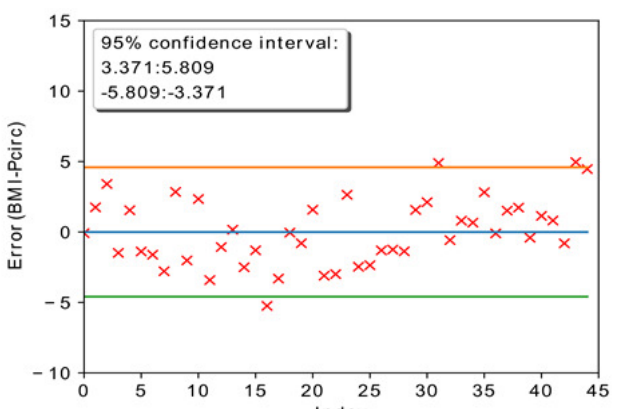
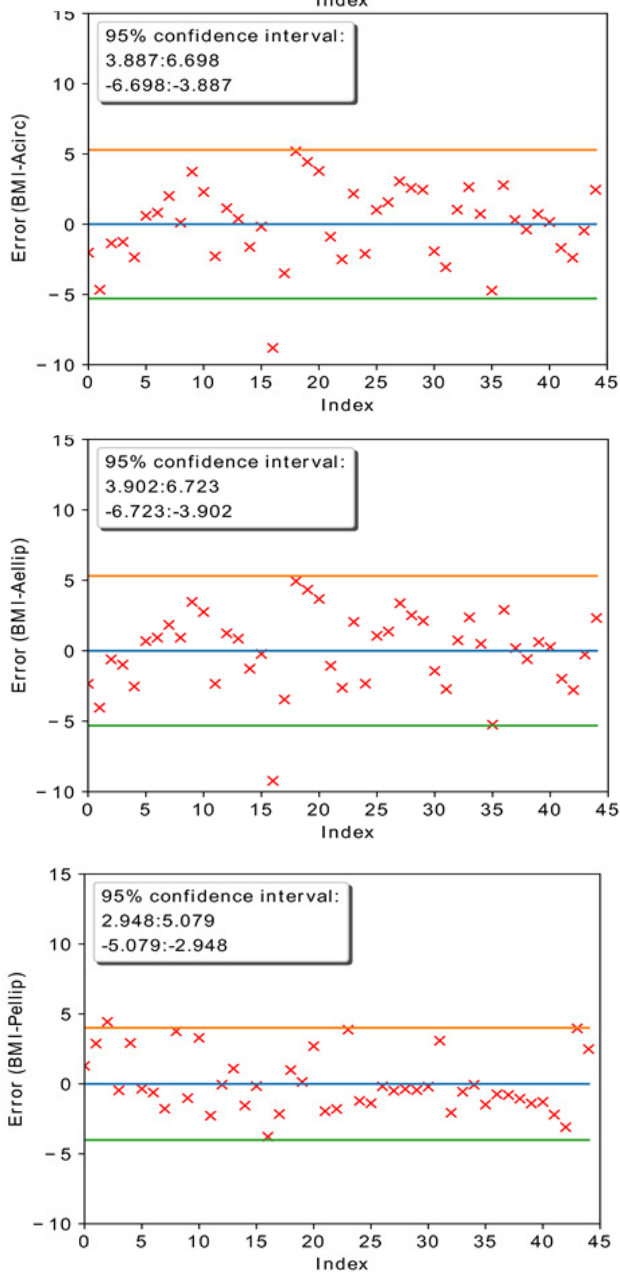

Fig. 4. Correlation analysis and Bland-Altman plots of parameters derived from sagittal plane image panel (a) Pcirc versus BMI panel, (b) Acirc versus BMI panel, (c) Pellip versus BMI panel, and (d) Aellip versus BMI.

MUAC was measured, and the correlation and the measurement agreement were evaluated against BMI. Compared to SMT, the result shows that the SMT measurements agreement is better than the MUAC's, given that the corresponding correlation coefficient is slightly lower than that of the upper arm circumference. Finally, the receiver operating characteristics of both SMT and MUAC were evaluated. The results show a slightly higher AUC area of 0.973 for MUAC compared to 0.966 for SMT. Moreover, the sensitivity was $100 \%$ for MUAC and $94.7 \%$ for SMT. However, the specificity was higher for SMT with a value of
$88.5 \%$ compared to $84.7 \%$ for MUAC. This shows that SMT performs better than MUAC since it gives excellent sensitivity without compromising for specificity. The current data are based on healthy subjects without any health issues except obesity. Therefore, special attention should be given when applying the proposed approach on people who are suffering from inflammations, metabolic disorders, stress, and cardiovascular dysfunctions, which may cause local changes in the shapes of specific areas of the human body, such as the lower and upper extremities, due to fat accumulation. 


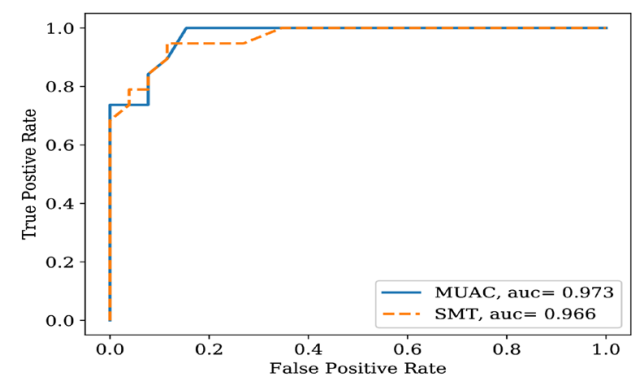

Fig. 5. Receiver operating characteristics of SMT and MUAC.

(a)

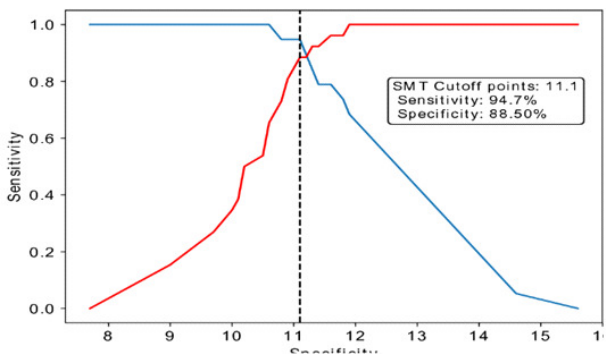

(b)

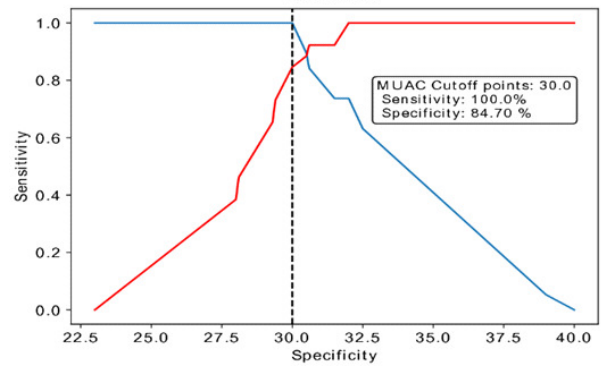

Fig. 6. (a) Optimal cutoff SMT and (b) optimal cutoff of MUAC.

Estimation of the BMI and the nutritional status using a sagittal plane image of the upper arm provides an indirect, easy, fast, and low-cost method. This method can be used in cases of disabilities, where it is difficult to perform traditional measurements of height and weight. Furthermore, it provides a fast way to find BMI whenever the needed instruments are not available or in temporary refugee camps. The technique used in the current research can be further investigated using automated image processing calculations. Using an automated system can make easier to build a massive database of varying population to be used in determining the nutritional status of the public. Moreover, this technique eliminates instrumentation errors and variability due to different operators.

\section{REFERENCES}

[1] M. M. Finucane, G. A. Stevens, M. J. Cowan, G. Danaei, J. K. Lin, C. J. Paciorek, G. M. Singh, H. R. Gutierrez, Y. Lu, A. N. Bahalim, F. Farzadfar, L. M. Riley, M. Ezzati, "National, regional, and global trends in body-mass index since 1980: Systematic analysis of health examination surveys and epidemiological studies with 960 country-years and 9.1 million participants", Lancet, Vol. 377, No. 9765, pp. 557-567, 2011

[2] M. Marmot, T. Atinmo, T. Byers, J. Chen, T. Hirohata, A. Jackson, W. P. T. James, L. Kolonel, S. Kumanyika, C. Leitzmann, J. Mann, H. J. Powers, K. S. Reddy, E. Riboli, J. A. Rivera, A. Schatzkin, J. C. Seidell, D. E. G. Shuker, R. Uauy, W. C. Willett, S. H. Zeisel, Food, Nutrition,
Physical Activity, and the Prevention of Cancer: A Global Perspective, American Institute for Cancer Research, 2007

[3] D. P. Guh, W. Zhang, N. Bansback, Z. Amarsi, C. L. Birmingham, A. H. Anis, "The incidence of co-morbidities related to obesity and overweight: A systematic review and meta-analysis", BMC Public Health, Vol. 9, No. 1, 2009

[4] G. Whitlock, S. Lewington, P. Sherliker, R. Clarke, J. Emberson, J. Halsey, N. Qizilbash, R. Collins, R. Peto, "Body-mass index and cause specific mortality in 900,000 adults: Collaborative analyses of 57 prospective studies", Lancet, Vol. 373, No. 9669, pp. 1083-1096, 2009

[5] F. Q. Nuttall, "Body mass index: Obesity, BMI, and health: A critical review", Nutrition Today, Vol. 50, No. 3, pp. 117-128, 2015

[6] A. Keys, F. Fidanza, M. J. Karvonen, N. Kimura, H. L. Taylor, "Indices of relative weight and obesity", International Journal of Epidemiology Vol. 43, No. 3, pp. 655-665, 2014

[7] World Health Organization, Physical Status: The Use and Interpretation of Anthropometry, Technical Report Series, Vol. 854, WHO, 1995

[8] K. M. Flegal, M. D. Carroll, R. J. Kuczmarski, C. L. Johnson, "Overweight and obesity in the United States: Prevalence and trends, 1960-1994", International Journal of Obesity and Related Metabolic Disorders, Vol. 22, No. 1, pp. 39-47, 1998

[9] J. S. Garrow, Treat Obesity Seriously: A Clinical Manual, Churchill Livingstone, 1981

[10] World Health Organization, Obesity: Preventing and Managing the Global Epidemic, Technical Report Series, Vol. 894, WHO, 2000

[11] R. T. Benn, "Some mathematical properties of weight-for-height indices used as measures of adiposity", British Journal of Preventive and Social Medicine, Vol. 25, No. 1, pp. 42-50, 1971

[12] J. Wang, J. C. Thornton, M. Russell, S. Burastero, S. Heymseld, R. N. Pierson, "Asians have lower body mass index (BMI) but higher percent body fat than do whites: Comparisons of anthropometric measurements", The American Journal of Clinical Nutrition, Vol. 60, No. 1, pp. 23-28, 1994

[13] C. L. Ogden, C. D. Fryar, M. D. Carroll, K. M. Flegal, "Mean body weight, height, and body mass index, United States 1960-2002", Advance Data, Vol. 347, pp. 1-17, 2004

[14] A. Zajacova, J. B. Dowd, S. A. Burgard, "Overweight adults may have the lowest mortality-do they have the best health?", American Journal of Epidemiology, Vol. 173, No. 4, pp. 430-437, 2011

[15] T. Sultana, M. N. Karim, T. Ahmed, M. I. Hossain, "Assessment of Under Nutrition of Bangladeshi Adults Using Anthropometry: Can Body Mass Index be Replaced by Mid-Upper-Arm-Circumference?", PloS One, Vol. 10, No. 4, Article ID e0121456, 2015

[16] P. Nguyen, U. Ramakrishnan, B. Katz, I. G. Casanova, A. E. Lowe, H. Nguyen, H. Pham, T. Truong, S. Nguyen, R. Martorell, "Mid-upper-arm and calf circumferences are useful predictors of underweight in women of reproductive age in northern Vietnam", Food and Nutrition Bulletin, Vol. 35, No. 3, pp. 301-311, 2014

[17] T. Khadivzadeh, "Mid upper arm and calf circumferences as indicators of nutritional status in women of reproductive age", Eastern Mediterranean Health Journal, Vol. 8, No. 4-5, pp. 612-618, 2002

[18] R. Chakraborty, K. Bose, S. Koziel, "Use of Mid-Upper Arm Circumference in Determining Undernutrition and Illness in Rural Adult Oraon Men of Gumla District, Jharkhand, India", Rural Remote Health, Vol. 11, No. 3, Article ID 1754, 2011

[19] V. A. Leandro-Merhi, J. Luiz Braga de Aquino, J. Gonzaga Teixeira de Camargo, "Agreement between body mass index, calf circumference, arm circumference, habitual energy intake and the MNA in hospitalized elderly", Journal of Nutrition Health and Aging, Vol. 16, No. 2, pp. 128132,2012

[20] J. M. Bland, D. G. Altman, "Statistical methods for assessing agreement between two methods of clinical measurement", Lancet, Vol. 1, No. 8476 , pp. 307-310, 1986

[21] D. Giavarina, "Understanding Bland Altman analysis", Biochemia Medica, Vol. 25, No. 2, pp. 141-151, 2015 